\title{
Hemofilia adquirida
}

Jaime García-Chávez y Abraham Majluf-Cruz *

${ }^{1}$ Servicio de Hematología; '2Unidad de Investigación Médica en Trombosis, Hemostasia y Aterogénesis. Instituto Mexicano del Seguro Social, Centro Médico Nacional La Raza, Hospital de Especialidades "Dr. Antonio Fraga Mouret", Ciudad de México, México

\section{Resumen}

La hemofilia adquirida (HA) es un trastorno hemostático autoinmune ocasionado por autoanticuerpos dirigidos contra el factor VIII:C. En 52 \% de los casos, la causa se desconoce o no se asocia con otra entidad patológica; en el resto, existen factores concomitantes: lupus, artritis reumatoide, cáncer, embarazo y medicamentos. En México no existe registro ni conciencia de la enfermedad entre el personal de salud. Los grupos de mayor incidencia son las mujeres en edad reproductiva y los individuos mayores de 70 años. Se caracteriza por hemorragia grave, sobre todo posterior a traumatismos y parto o cesárea, y equimosis grandes en tronco y extremidades. La sospecha es simple, basta que concurran hemorragia súbita, grave y un TTPa prolongado que no se corrige con plasma. El tratamiento consiste en lograr la hemostasia y erradicar el anticuerpo; lo primero se logra con el factor VII activado recombinante o concentrado del complejo de protrombínico activado. La ciclofosfamida, prednisona o rituximab sirven para erradicar el anticuerpo. La mayoría de los casos no son diagnosticados y la mortalidad es alta. Ya que los médicos desconocen el problema, no se sospecha, no se diagnostica y no se trata. Este documento revisa los datos más recientes de la HA y abunda en el diagnóstico y tratamiento.

PALABRAS CLAVE: Hemofilia. Hemofilia adquirida. Factor VIII. Autoinmunidad. Autoanticuerpos.

\section{Acquired hemophilia}

\begin{abstract}
Acquired hemophilia $(A H)$ is an autoimmune hemostatic disorder mediated by autoantibodies directed against factor VIII:C. In $52 \%$ of cases, the cause is unknown or is not associated with other pathological entities; in the rest, there are concomitant factors: lupus, rheumatoid arthritis, cancer, pregnancy, and medications. In Mexico, there is not a registry of $\mathrm{AH}$, and awareness of the disease among health personnel is low. The groups with the highest incidence are women of childbearing age and individuals older than 70 years. It is characterized by severe bleeding, especially after trauma and normal childbirth or cesarean delivery, and large ecchymoses in the trunk and extremities. The suspicion is simple, it just takes for sudden, severe hemorrhage and a prolonged activated partial thromboplastin time that is not corrected with plasma to concur in an individual. Treatment involves achieving hemostasis and eradicating the antibody. The former is achieved with recombinant activated factor VII or activated prothrombin complex concentrate. Cyclophosphamide, prednisone or rituximab are used to eradicate the antibody. Most cases of $\mathrm{AH}$ are not diagnosed, which translates into a high mortality rate. Given that awareness about the disease among physicians is low, it is not suspected, neither diagnosed, and nor is it treated. This document reviews the most recent data on $\mathrm{AH}$ and expands on its diagnosis and treatment.
\end{abstract}

KEY WORDS: Hemophilia. Acquired hemophilia. Factor VIII. Autoimmunity. Autoantibodies.

Correspondencia:

*Abraham Majuf-Cruz

E-mail: amajlufc@gmail.com
Gac Med Mex. 2020;156:67-77

Disponible en PubMed www.gacetamedicademexico.com

0016-3813/๑ 2019 Academia Nacional de Medicina de México, A.C. Publicado por Permanyer. Éste es un artículo open access bajo la licencia CC BY-NC-ND (http://creativecommons.org/licenses/by-nc-nd/4.0/). 


\section{Introducción}

La hemofilia adquirida $(\mathrm{HA})$ es un trastorno hemostático derivado del desarrollo de autoanticuerpos (auto-Ac) dirigidos principalmente contra el factor VIII:C (FVIII coagulante). Estos anticuerpos ocasionan hemorragias de un riesgo alto de morbilidad y mortalidad. En esta revisión, la mayoría de los conceptos se refieren a $\mathrm{HA}$ ocasionada por auto-Ac anti-FVIII, aunque existen casos de otros factores: V, VII, IX, X, XII y XIII. ${ }^{1}$

La HA tiene un sustento autoinmune. Casi siempre aparece por la producción descontrolada, sin causa aparente, de auto-Ac tipo IgG capaces de neutralizar el efecto del FVIII; por ello, a estos auto-Ac también se les llama "inhibidores". A diferencia de la hemofilia A hereditaria, en la cual el inhibidor es un alo-Ac anti-FVIII inducido por el factor aplicado, en la HA el auto-Ac aparece sin exposición al FVIII² (Tabla 1).

En $52 \%$ de los casos de HA no existen enfermedades concomitantes, sin embargo, en el resto coexiste con otras patologías:

- Autoinmunes como lupus eritematoso sistémico, artritis reumatoide, esclerosis múltiple, pénfigo, enfermedad inflamatoria intestinal crónica, asma o reacciones alérgicas graves.

- Linfoproliferativas y tumores sólidos (linfoma, leucemia, macroglobulinemia, próstata y pulmón).

- Primíparas en los primeros tres meses de puerperio $^{3}$ (Figura 1).

\section{Epidemiología}

La incidencia de la HA es muy baja. La literatura se limita a series de casos, registros y experiencias unicéntricas. Se estima 1.3 a 1.5 casos/millón de personas/año, ${ }^{4}$ por lo que se considera una enfermedad "huérfana", aunque es probable que haya muchos casos no diagnosticados. ${ }^{3}$ En México no existe registro, pero podemos hacer inferencias con base en la experiencia del Centro Médico Nacional La Raza del Instituto Mexicano del Seguro Social, cuya área de influencia es de tres millones de habitantes y solo se registraron 13 casos en 30 años (incidencia calculada de 0.43 pacientes/año). Sin embargo, este es un centro de referencia, por lo que el subdiagnóstico de la enfermedad parece evidente. Existe un gráfico bimodal en la incidencia. El primer pico corresponde a mujeres fértiles entre $19 \mathrm{y}$ 40 años; el segundo ocurre en adultos mayores (mediana de edad de 73.9 años, rango de 64 a 78), con predominio en varones (1.4:1) ${ }^{4,5}$ (Figura 2).

\section{Aspectos inmunológicos}

La HA ocurre por la producción de lgG1 e IgG4, policlonales, de cadenas ligeras kappa, dirigidas contra "hot spots" de los dominios A1, A2 y C2 del FVIII. Aunque se pueden generar auto-Ac contra cualquier factor hemostático, las características del FVIII lo hacen blanco preponderante. No es claro por qué se pierde la tolerancia al FVIII, pero se argumentan factores genéticos y ambientales. ${ }^{2}$ Se sugiere predisposición genética (genes de respuesta inmunológica y genotipo del HLA) por la asociación entre HA y polimorfismos del antígeno 4 de linfocitos T citotóxicos (CTLA-4 + 49 G) y expresión de los alelos DRB1*16 y DQB1*050210 del HLA clase II. ${ }^{2}$ Existe un equilibrio anormal de las células TCD4 + Th1 y Th2, lo cual permite producir el auto-Ac. El predominio de la subclase de IgG inducida por células T CD4-Th2 se asocia con concentraciones mayores de auto-Ac y peor pronóstico; cuando predominan las Th1, aumenta la respuesta al tratamiento inmunosupresor. ${ }^{6}$

Los auto-Ac asociados con HA inhiben la función del FVIII o aumentan su depuración, condicionando una caída del efecto hemostático. En la Figura 3 se muestra un diagrama de la molécula de este factor. Al reaccionar contra el FVIII, los auto-Ac inhiben su interacción con el FIX activado y con el FX (dominios A2-A3). Esta inhibición altera la generación de trombina y fibrina. El dominio C2 es en el cual el FVIII se une al FVW y a fosfolípidos, de manera que si este dominio es intervenido, la estabilización que proporciona el FVW al FVIII se modifica, lo cual acorta la vida media de este último. ${ }^{7}$

\section{Cuadro y diagnósticos clínicos}

Al cuadro lo caracterizan dos elementos. El primero es clínico, dado generalmente por equimosis extensas típicas, hemorragia en mucosas o, incluso, hemorragia interna en pacientes sin historia hemorrágica. Casi siempre, la HA se presenta abrupta, grave y agresivamente en pacientes previamente sanos. Con menos frecuencia existe una patología de base o primaria agudizada por hemorragia o síndrome anémico por la pérdida hemática. El segundo elemento es paraclínico dado por alargamiento aislado del tiempo de tromboplastina parcial activada (TTPa) que no se corrige al mezclar con plasma normal. La HA se sospecha en pacientes sin historia hemorrágica, con o sin hemorragia aparente y quienes, sin recibir anticoagulantes, tienen prolongado, aislado e inexplicable de TTPa. La sospecha aumenta si el paciente tiene algún factor de riesgo: enfermedad autoinmune, cáncer, edad mayor de 60 años y embarazo o 
Tabla 1. Principales diferencias entre hemofilia adquirida y hereditaria

\begin{tabular}{l|l}
\hline Hemofilia adquirida & Hemofilla hereditaria \\
\hline $\begin{array}{l}\text { Principalmente adultos mayores } \\
\text { No tiene un patrón hereditario }\end{array}$ & Habitualmente en la infancia \\
\hline Relación hombres:mujeres de 1:1 & Patrón de herencia ligada al cromosoma X \\
Hemartrosis raras & La mayoría son hombres \\
\hline $\begin{array}{l}\text { Auto-Ac con cinética tipo } 2 \\
\text { Sin correlación entre la concentración de FVIII:C. y la gravedad de la } \\
\text { hemorragia }\end{array}$ & Hemartrosis común \\
\hline $\begin{array}{l}\text { Mortalidad elevada } \\
\text { Tomado de Webert KE. Acquired hemophilia A. Semin Thromb Hemost 2012;38:735-741. }\end{array}$ & $\begin{array}{l}\text { Correlación entre la concentración de FVIII:C. y la gravedad } \\
\text { de la hemorragia }\end{array}$ \\
\hline
\end{tabular}

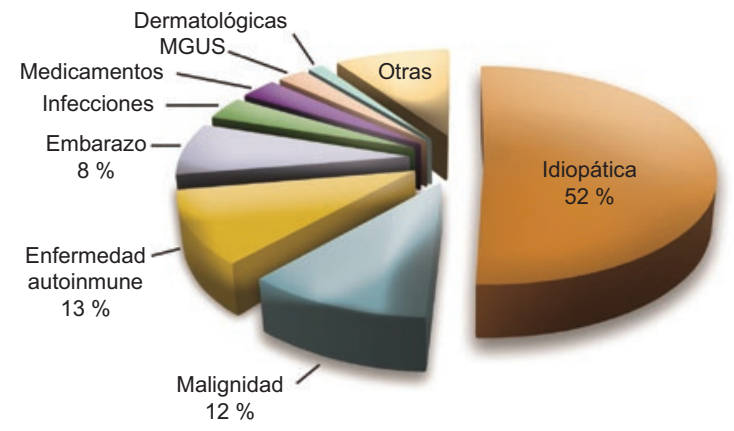

Figura 1. Distribución de la hemofilia adquirida de acuerdo con su naturaleza primaria o secundaria (tomado de referencia 15).

puerperio. Otras pruebas hemostáticas (plaquetas, tiempo de protrombina, fibrinógeno y tiempo de trombina) generalmente están normales. Debe descartarse HA en pacientes sin hemorragia aparente, pero con TTPa prolongado que no corrige con plasma normal y no tiene el anticoagulante lúpico positivo. ${ }^{8}$

Casi $94 \%$ de los pacientes tiene hemorragia, la cual se asocia con una mortalidad de 9 a $33 \% .^{7}$ La hemorragia aparece por estímulos irreconocibles (espontánea) 0 es postraumática. Las hemorragias graves representan 80 a $90 \%$ de los eventos y los sitios más frecuentes son sistema nervioso central, gastrointestinal, próstata, retroperitoneo y pulmón. A veces, la hemorragia es provocada por intervenciones diagnósticas o terapéuticas. La muerte por hemorragia generalmente ocurre en las primeras semanas de evolución; la tardía se asocia con la enfermedad primaria y el tratamiento inmunosupresor. ${ }^{4}$ Aunque la HA es una enfermedad rara con un riesgo alto de morbilidad y mortalidad, la experiencia clínica es limitada porque la mayor evidencia proviene de casos aislados. ${ }^{9}$ Cuando la HA está asociada con una patología primaria, las características de esta imprimen su huella clínica.
En servicios de contacto inicial (urgencias, ginecoobstetricia, medicina familiar, medicina interna y oncología), ${ }^{10}$ la enfermedad no se sospecha y se indican maniobras invasivas para la atención integral, aunque siendo un problema hemostático, resultan contraproducentes ya que aumentan el riesgo y la intensidad de la hemorragia o llevan la situación a estados críticos por hemorragia masiva o en órganos vitales. El diagnóstico final suele ser tardío (hasta 30 días de retraso). ${ }^{8}$ La responsabilidad es grande porque debe tenerse un índice de sospecha alto para establecer manejo temprano que mejore el pronóstico, ya que el tratamiento agudo casi siempre recae en estos especialistas mientras que la fase estable y de resolución definitiva depende más del hematólogo.

A diferencia de la hemofilia congénita, la HA cursa con hematomas grandes, difusos, dolorosos que pueden generar anemia. Otras manifestaciones incluyen hematomas musculares y en mucosas gastrointestinal y genitourinaria. La hemorragia también complica la situación del paciente posoperado o con heridas. Puede haber hemorragia retroperitoneal, 9,11 pero la articular o intracraneal es menos frecuente. ${ }^{11}$ La hemorragia muscular puede generar pérdida hemática importante $y$, si no es tratada adecuada y prontamente, ocasionar isquemia vascular o nerviosa por compresión. ${ }^{12}$ Los hematomas grandes en piernas pueden diagnosticarse erróneamente como trombosis venosa profunda: ${ }^{12}$ si se indican antitrombóticos inducirán hemorragias masivas. La formación de abscesos es otra complicación si el tratamiento se retrasa. La hemorragia muscular profunda, como en el psoas, es difícil de diagnosticar y se confunde con abdomen agudo, indicativo de cirugía subsecuente. Cualquier cirugía es compleja por el riesgo hemorrágico alto y puede condicionar la, incluso extracciones dentales, biopsias o colocación de catéteres centrales. 


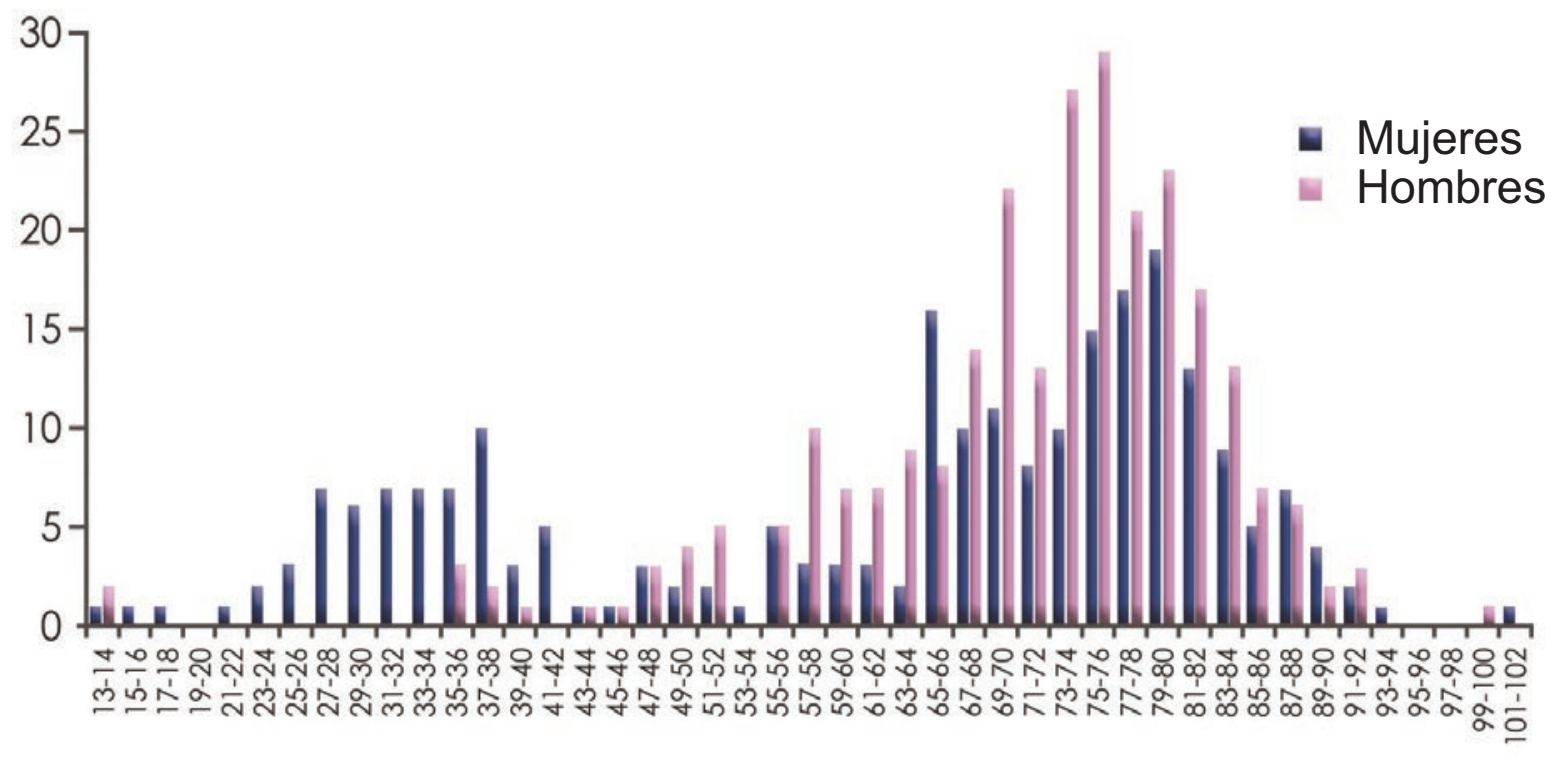

Figura 2. Distribución de casos de hemofilia adquirida del registro europeo EACH2. ${ }^{3}$

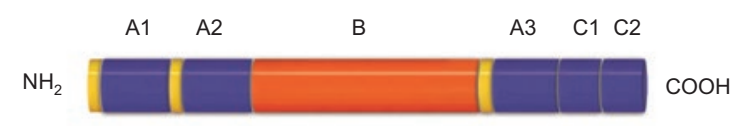

Figura 3. Dominios del FVIII humano.

Las pruebas de escrutinio o confirmatorias pueden interpretarse erróneamente. Toda coagulopatía hereditaria leve o moderada puede pasar desapercibida pero manifestarse tardíamente como hemorragia. Esto se descarta por anamesis y corrección de las pruebas hemostáticas al mezclar el plasma del paciente con plasma normal. La corrección sugiere una deficiencia de factores y descarta un inhibidor. Otra condición que confunde es el anticoagulante lúpico, auto-Ac asociado con TTPa alargado y patrón de no corrección con plasma normal. Existe diferencia entre el auto-Ac de la $\mathrm{HA}$, el cual requiere tiempo y temperatura para manifestarse completamente y el anticoagulante lúpico, el cual no requiere ni tiempo ni temperatura, además de asociarse con trombosis. ${ }^{13}$ Un escenario muy complicado es cuando conviven ambos auto-Ac. Si bien en el diagnóstico diferencial debe considerarse la coagulación intravascular diseminada, esta se descarta si el coagulograma arroja un perfil de deficiencia múltiple de factores, además de datos de consumo como el aumento de dímero $\mathrm{D}$ y trombocitopenia. ${ }^{14}$

\section{Hemofilia adquirida y embarazo}

La sintomatología es heterogénea en presentación e intensidad. La hemorragia es siempre moderada 0 grave y en $50 \%$ no existe factor desencadenante. ${ }^{15}$
Hasta $75 \%$ de HA asociada con el embarazo ocurre en primigrávidas. ${ }^{16}$ Las hemorragias son casi siempre subcutáneas, mucosas o retroperitoneales y las hemartrosis muy raras. Ocasionalmente, la hemorragia aparece hasta seis semanas luego del nacimiento. La HA raramente complica el embarazo; tiene un pronóstico mejor porque ocurren remisiones espontáneas (incluso, el auto-Ac materno puede desaparecer sin tratamiento) y el índice de mortalidad es más bajo (hasta $6 \%$ ). ${ }^{17,18}$ Cuando el seguimiento prenatal es adecuado, es factible diagnosticar HA con el TTPa como parte de los preoperatorios o si aparece hemorragia leve, evaluando si el TTPa está prolongado aisladamente. Hasta un tercio de las pacientes no requiere tratamiento, ${ }^{19} \sin$ embargo, en todo momento pueden aparecer hemorragias graves hasta erradicar al inhibidor. ${ }^{17}$

Existen casos de transferencia in utero del inhibidor al producto, aunque la hemorragia en el niño es inusitada..$^{18}$ Cuando el producto depura el auto-Ac materno el cuadro clínico desparece si es que se presentó; no hay posibilidad de que vuelva este problema. La manera más efectiva y menos costosa de vigilar al niño es con el TTPa, el cual se acorta hasta normalizarse en máximo seis semanas.

\section{Diagnóstico por laboratorio}

El estudio se inicia con el TTPa, prueba simple y disponible en casi cualquier hospital, rápida, estandarizada y económica. En servicios de urgencias, todo TTPa prolongado debería ser estudiado siempre. Este 


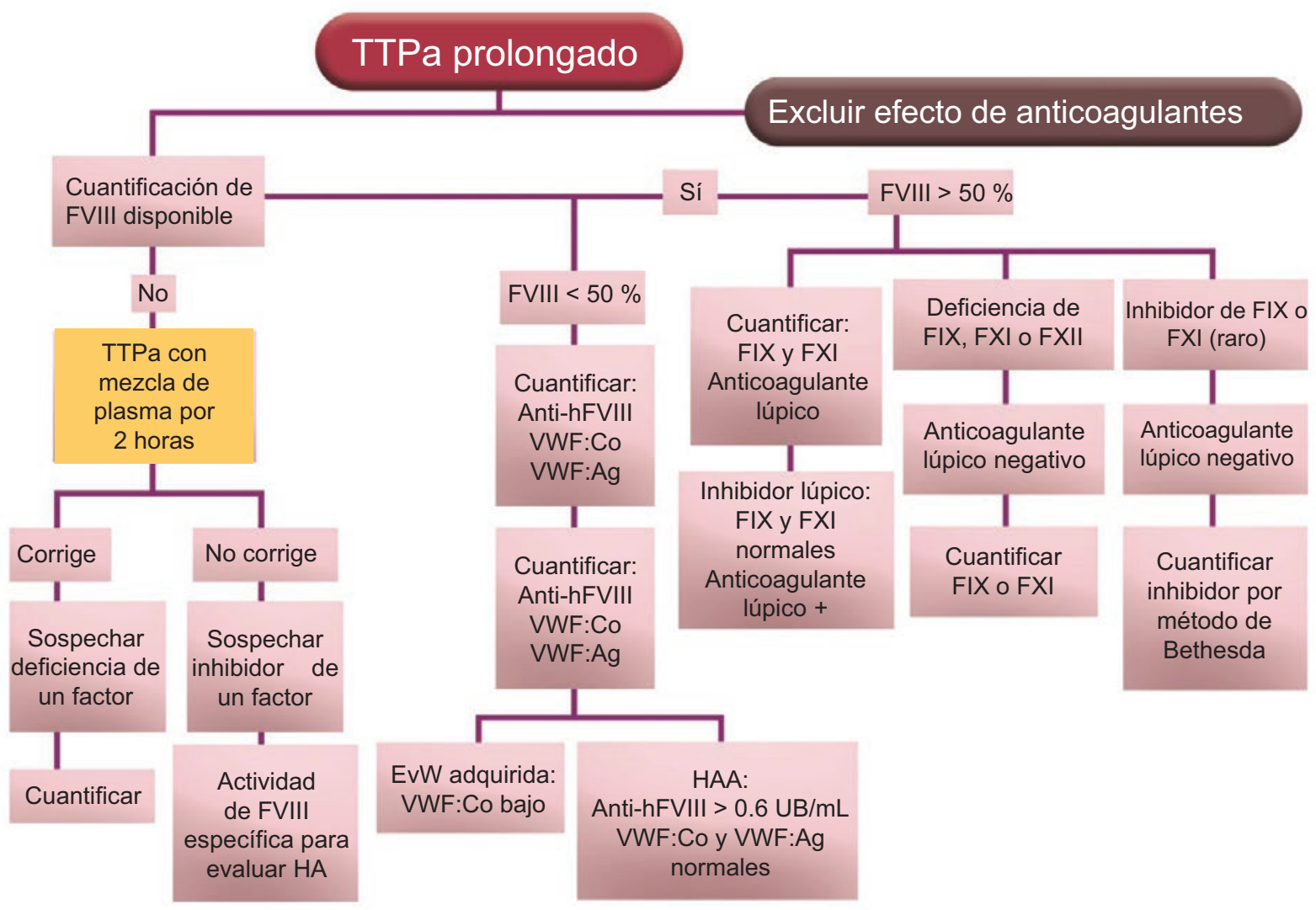

Figura 4. Manejo del paciente con el tiempo de tromboplastina parcial activada prolongado.

se prolonga en relación con el testigo normal y no se corrige al adicionar una mezcla de plasmas (también Ilamada pool o testigo), lo cual sugiere la presencia de un inhibidor que bloquea el flujo de reacciones enzimáticas reflejadas en el TTPa (Figura 4). Casi siempre, el TTPa está prolongado aisladamente y si es así, debe interrogarse rigurosamente para buscar datos de hemorragia.

El siguiente paso para el diagnóstico por laboratorio es medir el FVIII:C. funcional, el cual se encontrará debajo del rango normal. El tercer paso y definitivo es cuantificar la potencia del inhibidor mediante las pruebas de Bethesda o Nijmegen-Bethesda, las cuales evalúan el título del inhibidor en unidades Bethesda (UB). La variación de estas pruebas es alta, lo cual se traduce en resultados falsos positivos y negativos. Existe una prueba de ELISA para estos auto-Ac anti-FVIII, aunque también arroja resultados falsos negativos. ${ }^{20,21}$ Otras pruebas más específicas son complejas, caras y poco accesibles en la mayoría de los hospitales. ${ }^{22}$

Existen varias técnicas de medición del FVIII:C. y su inhibidor, funcionales y antigénicas. Entre las primeras hay dos clases: coagulométricas y cromogénicas. La técnica de ELISA se usa generalmente para las antigénicas. Las cromogénicas tienen ventajas: son técnicamente robustas, fáciles de usar y el anticoagulante lúpico no interfiere. Sus inconvenientes son su costo y la interferencia de heparinas, lipemia, hemólisis y anticoagulantes orales directos. Esta técnica suele dar resultados más bajos de FVIII:C. que la coagulométrica, lo que resuelve algunas inconsistencias de esta última. La técnica coagulométrica se basa en el TTPa y tiene ventajas indiscutibles: es la más utilizada y es automatizada, por lo que es la más recomendable. Tiene inconvenientes: la afectan la cantidad y calidad de los fosfolípidos empleados y requiere de activadores. Además, el nivel residual de FVIII:C. puede no ser tan preciso (la cuantificación de FVIII:C. puede ser 50 a $100 \%$ mayor que con la cromogénica). Cualquier técnica para medir el inhibidor tiene variación. Una vez elegido un método, la vigilancia de la enfermedad debe ser con el mismo.

$\mathrm{El}$ inhibidor debe evaluarse mediante el método Bethesda, que mide la cantidad residual del FVIII:C. en una mezcla de pool de plasma y plasma del paciente diluidos seriadamente $(1: 2,1: 4,1: 8$ y así sucesivamente), incubados 2 horas a $37^{\circ} \mathrm{C} .{ }^{23} \mathrm{EI} \mathrm{FVIII} \mathrm{aumenta} \mathrm{con}$ la dilución. La relación de la cuantificación del 
inhibidor (1.0 UB) es recíproca con el valor de la dilución del plasma del paciente que permite $50 \%$ de inhibición. Así, 1 UB es la cantidad del inhibidor que neutraliza $50 \%$ del FVIII:C. circulante (2 UB neutralizan $75 \%$, 3 UB 87.5 y así sucesivamente).

La modificación Nijmegen es más precisa si el inhibidor es muy bajo; se adiciona un buffer a la prueba Bethesda para mantener el plasma control en $\mathrm{pH}$ fisiológico 2-3 horas y permitir la estabilización del FVIII. ${ }^{21}$

Aunque estas pruebas son útiles en hemofilia hereditaria, la determinación del auto-Ac en HA es más difícil por su cinética tipo II (ver adelante). Idealmente debe medirse la concentración del auto-Ac unido a FVIII para evaluar mejor al inhibidor ${ }^{24}$ mediante ELISA, gel de agarosa, inmunoprecipitación o con microperlas fluorescentes. ${ }^{25}$ Este último método parece mejor porque no es afectado por el FVIII:C residual, el anticoagulante lúpico o la heparina.

Los inhibidores del FVIII de la HA y de la hemofilia A hereditaria son diferentes (Figura 5). Los alo-Ac de la hemofilia hereditaria son inhibidores tipo I (cinética de primer grado), inhiben al FVIII en relación lineal con su concentración e inhiben totalmente al factor en concentraciones altas. La actividad residual del FVIII:C cae con el tiempo de incubación, en forma saturada y en línea recta hasta que FVIII:C. es cero. Es decir, la inactivación por el alo-Ac es lineal y dependiente de la temperatura y el tiempo. Los auto-Ac de la HA son no lineales (tipo II), inicialmente inhiben rápido pero luego se equilibran y puede encontrarse actividad residual del FVIII, aún luego de incubarse con concentraciones máximas del inhibidor por un periodo adecuado. Es decir, el inhibidor de la HA condiciona una caída inicial recta, como cinética tipo I, pero después se comporta como meseta y la concentración de FVIII:C. residual no disminuye a cero (Figura 5). ${ }^{17}$

La actividad residual no se correlaciona ni con el título del inhibidor ni con la tendencia hemorrágica. En la HA, el nivel del FVIII:C. residual no se interpreta como en la hemofilia hereditaria. Los pacientes con HA y FVIII:C residual de $20 \%$ se asemejan a los pacientes con hemofilia hereditaria con una concentración de FVIII muy inferior; ${ }^{13}$ el paciente con HA tiene hemorragias más graves que aquel con hemofilia congénita con niveles comparables de FVIII:C. Incubar FVIII en exceso no neutraliza al inhibidor tipo II in vitro y las dosis altas de FVIII son ineficaces en HA si el inhibidor es muy alto, dificultando el tratamiento.

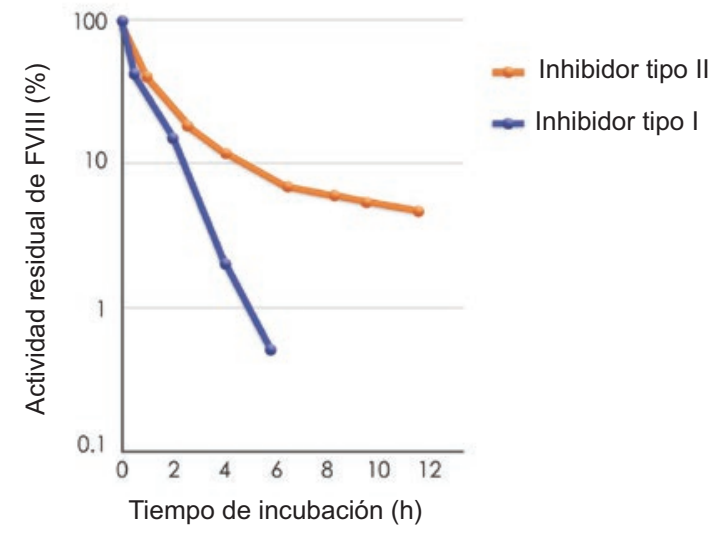

Figura 5. Cinética de los inhibidores tipos 1 y 2.

Existen errores de medición y de interpretación. Para los primeros, es importante recordar que el inhibidor es un auto-Ac que se hace evidente incubando a $37^{\circ} \mathrm{C}$; si esto se omite, el resultado se confunde con anticoagulante lúpico o con efecto de heparina $u$ otros anticoagulantes. En la interpretación, si el médico solicita la medición coagulométrica de otros factores hemostáticos (FIX, FXI o FX) porque el TTPa está prolongado, los niveles estarán bajos ya que la medición de factores se basa en el TTPa el cual está afectado por el inhibidor del FVIII; el resultado es falso positivo diagnosticando erróneamente deficiencia múltiple de factores. Ya que es infrecuente que un anticuerpo se dirija contra varios factores, la medición antigénica o funcional cromogénica aclara las cosas. Otro error de interpretación derivado de la cinética tipo II del auto-Ac de la HA es suponer que una dosis alta de FVIII:C. (15-30\%) protege al paciente; esto es válido para la hemofilia hereditaria (cinética tipo I), pero no para la HA en la cual un FVIII:C. de $20 \%$ puede asociarse con hemorragia grave.

En nuestra experiencia, el principal factor que retrasa el diagnóstico es la falta de conocimiento acerca de esta patología debido a su baja prevalencia y a que no se enseña durante la formación médica. Los médicos de primer contacto habitualmente no tienen información. Incluso en la residencia de hematología, la HA no se dimensiona como urgencia diagnóstica y terapéutica. Además, hacen falta laboratorios especializados en hemostasia que tengan un buen control de calidad, perfil que muchas veces tampoco cumplen los centros de tercer nivel.

\section{Complicaciones y pronóstico}

Retrasar el diagnóstico empeora el pronóstico. Este depende del curso de la enfermedad, el cual es 
paralelo a la gravedad hemorrágica y la enfermedad subyacente. Las remisiones completas "espontáneas" ocurren entre 25 a $36 \%$ de los casos, sobre todo asociadas con el embarazo y la administración de medicamentos. ${ }^{19,26}$ La supervivencia global es de 69 a $78 \%$ y, alcanzada la remisión, es similar a la de la población general o de la afectada por la enfermedad primaria. Hasta $8 \%$ muere pronto (mediana: 19 días posdiagnóstico), generalmente por hemorragia incontrolable..$^{19}$ La muerte en la primera semana posdiagnóstico es casi siempre por hemorragia gastrointestinal o pulmonar. La hemorragia intracraneal y retroperitoneal se asocia con mortalidad más tardía. ${ }^{27}$ La muerte se asocia con la HA, con la enfermedad concomitante o con efectos secundarios del manejo de la enfermedad primaria. La mortalidad por tratamiento de la enfermedad primaria es similar o mayor que la de hemorragia. ${ }^{16}$ La HA asociada con el embarazo es la excepción ya que la supervivencia llega a 13 meses en $100 \% .^{16}$

Los factores asociados con mal pronóstico para remisión y supervivencia son: FVIII:C. $<1 \mathrm{UI} / \mathrm{dL}$ al diagnóstico, puntuación $>2$ en la escala funcional de la Organización Mundial de la Salud, asociación con neoplasias y no alcanzar remisión completa. La edad mayor de 65 años al diagnóstico disminuye la supervivencia. ${ }^{4,8}$ Los factores de buen pronóstico son alcanzar remisión completa, tener actividad del FVIII > $1 \mathrm{UI} / \mathrm{dL}$ y un título de inhibidor $<16$ UB. ${ }^{8}$ En general, la HA es potencialmente curable con inmunosupresores o inmunomoduladores, los cuales erradican el auto-Ac.

Si se logra remisión, el monitoreo clínico y por laboratorio debe ser frecuente por el riesgo de recurrencia; $20 \%$ recae en los dos primeros años. ${ }^{8,28} \mathrm{El}$ título de inhibidor y del FVIII:C. se miden semanalmente mientras se indiquen inmunosupresores. Alcanzada la remisión, las evaluaciones son mensuales los primeros seis meses, cada dos o tres meses los siguientes seis meses y cada seis meses durante el seguimiento por dos años. ${ }^{1,29}$ Para la HA asociada con el embarazo, la recurrencia en embarazos subsecuentes es baja y la paciente debe informarse de esta posibilidad. La recaída se maneja con el tratamiento instituido inicialmente.

\section{Tratamiento}

Lograr un resultado favorable en la HA depende del diagnóstico expedito y la selección del mejor manejo. El tratamiento descansa en dos pilares:
- Controlar la hemorragia si está presente o prevenir su aparición.

- Erradicar el inhibidor. ${ }^{17}$

Ya que el paciente puede morir rápidamente, ${ }^{26}$ se debe indicar tratamiento hemostático pronto dependiendo de la gravedad y localización de la hemorragia. No deben realizarse procedimientos invasivos como evacuación de hematomas a menos que sean absolutamente necesarios, preferentemente hasta que el inhibidor haya sido erradicado, ${ }^{8}$ porque no hay prueba confiable para evaluar la respuesta coagulante y no puede garantizarse una hemostasia mínima. ${ }^{19}$ Muchas hemorragias que ponen en peligro la vida son provocadas por intervenciones médicas: punciones venosas, colocación de catéteres y sondas, toma de biopsias y otros procedimientos invasivos diagnósticos o de monitoreo, como tomar frecuentemente la presión arterial. ${ }^{30}$ Sugerimos evitar todo procedimiento invasivo que no sea estrictamente necesario. Si se requiere sin postergación, solo si el tratamiento hemostático ya está instalado. Esta acción simple reduce las hemorragias iatrógenas. ${ }^{31}$

El paciente debe ser informado respecto de la aparición de síntomas tempranos de hemorragia y de la prevención de traumatismos y evitar medicamentos especialmente analgésicos no esteroideos. Se debe enfatizar que busque atención en caso de hemorragia no justificada.

Entre 20 y $30 \%$ de los pacientes no requieren tratamiento hemostático al diagnóstico, por ejemplo si presenta equimosis extensas. Se debe intentar inmediatamente erradicar el inhibidor y, a menos que exista hemorragia evidente o descontrol hemodinámico por hipovolemia, no se inicia tratamiento hemostático de inmediato. Tampoco requieren tratamiento los pacientes con inhibidor y deficiencia funcional del FVIII:C. pero sin hemorragia. Los enfermos que deben recibir tratamiento hemostático inmediato son aquellos con riesgo hemorrágico alto o con hemorragia clínica 0 paraclínica: muscular, genitourinaria, gastrointestinal, retroperitoneal, articular, pulmonar o del sistema nervioso central. Si el paciente se somete a un procedimiento invasivo mayor o menor debe recibir tratamiento hemostático antes y después del mismo. ${ }^{28}$

Ya que el tratamiento está limitado por la disposición de recursos, las consideraciones a partir de este punto parecen utópicas, aunque es necesario conocer las opciones existentes. La intensidad del tratamiento hemostático se define por la localización y gravedad hemorrágicas. ${ }^{114}$ Aunque el tratamiento de elección es con agentes de puenteo y FVIII porcino recombinante, 
Tabla 2. Agentes hemostáticos de primera línea para hemofilia adquirida

\begin{tabular}{|c|c|c|}
\hline Agente & Dosis recomendada & Comentarios \\
\hline \multicolumn{3}{|c|}{ Terapia de reemplazo } \\
\hline rpFVIII & $\begin{array}{l}\text { - Si no existe inhibidor anti-rpFVIII: 50-100 U/kg } \\
\text { cada } 2 \text { a } 3 \text { horas y redosificar como se } \\
\text { necesite. } \\
\text { - Si existe inhibidor anti-rpFVIII: } 200 \text { U/kg para } \\
\text { hemorragia grave. } \\
\text { - 50-100 U/kg para hemorragia moderada. } \\
\text { - Redosificar como se necesite. }\end{array}$ & $\begin{array}{l}\text { - Primera línea de tratamiento (si hay), sobre todo en los } \\
\text { casos más graves. } \\
\text { - Puede monitorearse con TTPa. } \\
\text { - Reemplaza el componente deficiente. } \\
\text { - Eficacia probada. } \\
\text { - Menos efectivo si existen anticuerpos cruzados. } \\
\text { - Generación potencial de anticuerpos } \\
\text { - Requiere monitoreo estrecho. } \\
\text { - Primera línea si no hay anticuerpos anti-rpFVIII, existe } \\
\text { cuantificación de FVIII y la hemorragia pone en riesgo una } \\
\text { extremidad. }\end{array}$ \\
\hline
\end{tabular}

Agentes de puenteo

\begin{tabular}{|c|c|c|}
\hline $\begin{array}{l}\text { Concentrado de complejo } \\
\text { protrombínico activado }\end{array}$ & $\begin{array}{l}\text { - 50-100 U/kg cada } 8 \text { a 12/horas. } \\
\text { - No exceder } 200 \text { U/kg/día. }\end{array}$ & $\begin{array}{l}\text { - Eficacia probada. } \\
\text { - Sin pruebas para monitoreo. } \\
\text { - Potencial trombótico. } \\
\text { - Primera línea si no existe rpFVIII, existe Ac anti- } \\
\text { rpFVIII > } 10 \text { UB, no existe cuantificación de FVIII y en } \\
\text { hemorragia no grave. }\end{array}$ \\
\hline FVII recombinante activado & $\begin{array}{l}\text { - } 70-90 \text { yg cada } 2 \text { a } 3 \text { horas hasta lograr la } \\
\text { hemostasia. } \\
\text { - Posteriormente, dosificar como se necesite. }\end{array}$ & $\begin{array}{l}\text { - Eficacia probada. } \\
\text { - Sin pruebas para monitoreo. } \\
\text { - Vida media corta. } \\
\text { - Potencial trombótico. } \\
\text { - Primera línea si existe anticuerpo anti-rpFVIII > } 10 \text { UB, no } \\
\quad \text { hay cuantificación de FVIII, en hemorragia no grave o la } \\
\text { hemorragia pone en riesgo una extremidad. }\end{array}$ \\
\hline
\end{tabular}

Simultáneamente con el tratamiento debe iniciarse terapia inmunosupresora. La actividad del FVIII debe monitorearse continuamente. No existen estudios que comparen todas estas alternativas terapéuticas, por lo que la elección del agente se basa más en la disponibilidad, en la experiencia del médico y en cuestiones económicas. La eficacia hemostática se basa en la evaluación clínica y el cambio a otro agente debe considerarse luego de 12 a 24 horas. Una vez que se logra la hemostasia, la frecuencia de las dosis de cualquier tratamiento debe disminuirse tanto como sea posible para prevenir una trombosis. Estos tratamientos no deben indicarse si el nivel del FVIII está aumentando, si no existe riesgo elevado de hemorragia o si no existe hemorragia. Es muy importante considerar la profilaxis antitrombótica si el nivel de FVIII es mayor de $50 \%$. rpFVIII = factor VIII porcino recombinante.

si no se dispone de ellos y si el inhibidor es < 5 UB y se puede monitorear frecuentemente la recuperación de FVIII:C. (manteniéndolo $>5 \%$ ), basta con aportar oxígeno, soporte general y aumentar la concentración de FVIII con desmopresina. ${ }^{32}$ El ácido tranexámico se recomienda en algunas guías para hemorragia en mucosas. Si se utiliza concentrado de complejo protrombínico activado ( $\mathrm{CCPa})$, el ácido tranexámico nunca debe indicarse antes de 12 horas.

Desafortunadamente, la mayoría de las hemorragias en la HA son graves e incluso mortales, siendo la anemia otro factor que influye en el tratamiento. En la medida en que la hemorragia progresa, se requiere más terapia hemostática y monitoreo de su eficacia, evaluada mediante la mejoría clínica y el nivel de hemoglobina.

El tratamiento inicial de la hemorragia grave es con agentes de puenteo. El CCPa contiene los factores II, VII, IX y Xa capaces de puentear al FVIII para generar trombina $^{33}$ (Tabla 2). La dosis es de 50 a $100 \mathrm{UI} / \mathrm{kg}$ cada ocho a 12 horas o incluso 35 a $80 \mathrm{Ul} / \mathrm{kg}$ cada ocho a 24 horas $^{34}$ (nunca pasar de 200 Ul/kg/día); es útil en 76 a $89 \%$ de los casos. ${ }^{34}$ En pacientes con hemorragias crónicas y leves se usa profilácticamente $50 \%$ de la dosis. El FVII activado recombinante (rFVIla) permite puentear la hemostasia desde la vía extrínseca, evitando a la intrínseca. ${ }^{35,36}$ La evidencia del rFVlla como primera línea de tratamiento en HA es grande. Se emplean 90 a $120 \mu \mathrm{g} / \mathrm{kg}$ cada dos a tres horas y la dosis se disminuye según la respuesta., ${ }^{1,148}$ Su eficacia llega a $100 \%$ (81 a $100 \%$ ). ${ }^{1,37}$ La efectividad y seguridad es discretamente mayor con rFVIla que con CCPa, pero mayor con ambos que con FVIII o desmopresina (93.3 versus $68.3 \%, p=0.003) .{ }^{5}$ No hay estudios que hagan preferible a uno u otro. ${ }^{8,38}$ Debe recordarse que las dosis para HA se extrapolan de hemofilia hereditaria con inhibidor, que se desconoce la duración óptima del tratamiento, aunque puede mantenerse hasta controlar la hemorragia, que, empíricamente, se recomiendan dosis adicionales para prevenir la reaparición de hemorragia, ${ }^{14}$ y que no existen pruebas de laboratorio para monitorearlos (la evaluación clínica es la mejor herramienta de vigilancia). Finalmente, rFVIla y CCPa pueden administrarse alternadamente en hemorragias 
Tabla 3. Riesgo de trombosis en pacientes tratados con agentes de puenteo

\begin{tabular}{|l|l|c|c|}
\hline Estudio & Tratamiento & $n$ & $\%$ \\
\hline Summer $^{1}$ & rFVII & 139 & 2.9 \\
\hline Ingerslev $^{2}$ & Agentes combinados o alternados & 9 & 55 \\
\hline Baudo $^{3}$ & rFVII & 174 & 2.9 \\
\hline & CCPa & 63 & 4.8 \\
\hline Seita $^{4}$ & FVIII/desmopresina & 70 & 0 \\
\hline Borg $^{5}$ & rFVIII & 132 & 2.3 \\
\hline & CCPa & 28 & 0 \\
\hline Tiede $^{6}$ & rFVII & 6 & 0 \\
\hline & rFVII/ácido tranexámico & 63 & 5 \\
\hline
\end{tabular}

graves resistentes si la monoterapia se administra a dosis y frecuencia máximas. ${ }^{14,32,38}$ Es una opción en hospitalizados bajo tutela de un médico con experiencia en coagulopatías.

Los efectos adversos directos de los agentes de puenteo son escasos aunque justificables por la gravedad. La trombosis es la complicación más temida. Una buena cantidad de pacientes con HA tiene patologías primarias protrombóticas, por lo que es crucial respetar la dosis recomendada y detener el tratamiento al momento, principalmente en pacientes de edad avanzada con riesgo aterotrombótico alto y cáncer o mujeres posparto, es decir, con estados protrombóticos. Aunque la trombogenicidad tanto del aFVIIr como del CCPa es baja, la trombosis debe considerarse siempre ${ }^{5,39}$ (Tabla 3). Debido a la rareza de la $\mathrm{HA}$, no existe evidencia para indicar tromboprofilaxis o tratamiento antitrombótico. Este es un problema de toma de decisiones, ya que frecuentemente el paciente tiene factores de riesgo trombogénico tributarios de anticoagulación total pero es candidato para tratamiento hemostático potente para el manejo de HA. Existen solo recomendaciones de expertos que sugieren líneas tromboprofilácticas generales: uso de medias elásticas o compresión neumática intermitente en tanto el FVIII-C sea $<50 \%$ de actividad, ya que, una vez alcanzado este nivel, el paciente puede recibir tromboprofilaxis o anticoagulación terapéutica. ${ }^{5,36}$ Obviamente siempre se debe personalizar el tratamiento.

El monitoreo por laboratorio de la HA es difícil y por ello la respuesta clínica es la estrategia más aplicable. ${ }^{40}$ En el paciente con hemofilia $\mathrm{A}$ e inhibidor, calcular el efecto de de FVIII es hipotéticamente posible porque se trata de inhibidores tipo I. En la HA, el inhibidor es tipo II, lo cual dificulta evaluar la vigilancia por laboratorio. El problema es más evidente en pacientes con títulos altos de inhibidor (> $16 \mathrm{UB}$ ), por lo tanto, la alternativa más efectiva es monitorear frecuentemente el estado clínico y cuantificar el FVIII y el TTPa.

El siguiente paso terapéutico es buscar la curación erradicando el inhibidor con inmunosupresores. El paciente logra remisión completa si el inhibidor es $<0.6$ UB y el FVIII:C. es $>50 \% .{ }^{8}$ La recaída se define cuando al suspender el inmunosupresor, reaparece el inhibidor a títulos crecientes asociado con descenso progresivo del FVIII:C. ${ }^{5}$ La remisión estable es la persistencia de la remisión completa después de suspender la terapia.

Debe mencionarse que la opción ideal se desconoce. El tratamiento de primera línea más utilizado es la administración de esteroides ( $1 \mathrm{mg} / \mathrm{kg} / \mathrm{día})$, solos o asociados con ciclofosfamida en dosis bajas (1 a $2 \mathrm{mg} / \mathrm{kg}$ / día), por tres a cinco semanas ${ }^{1}$ (Tabla 4). La evidencia se basa en series pequeñas y retrospectivas. Solo dos estudios prospectivos pequeños mostraron respuestas de casi $60 \%$ con la combinación, mejor que con solo esteroides (42\%).. Otros registros muestran ventaja no significativa de la combinación sobre monoterapia con esteroides (80 versus $70 \%$, respectivamente). ${ }^{1}$

Aparentemente no hay diferencia significativa para la supervivencia global y libre de enfermedad entre los dos tratamientos. ${ }^{28}$ La inmunosupresión aunada a las comorbilidades del enfermo (edad, multipatología, desequilibrio actual) deriva en el riesgo de citopenias e infecciones secundarias (33 a $53 \%$ );: ${ }^{28}$ entre 11 y $12 \%$ de la mortalidad asociada con HA se atribuye a estas complicaciones. ${ }^{8}$ En la HA asociada con el embarazo, la ciclofosfamida puede tener efecto leucemógeno y quizá sobre la fertilidad. ${ }^{28}$ El tratamiento de segunda línea es rituximab (Tabla 4), de elección si el de primera línea falla. ${ }^{1,14,28} \mathrm{Se}$ cree que su efecto es por disminución del auto-Ac anti-FVIII. Se inicia a $375 \mathrm{mg} / \mathrm{m}^{2} / \mathrm{semana}$ por cuatro semanas $^{8}$ y se requieren semanas o meses. La erradicación se logra en $78.6 \%$ de los pacientes, independientemente del uso previo o no de otro inmunosupresor. ${ }^{42}$ Es una buena alternativa si el título del inhibidor es $>200$ UB, ${ }^{43}$ sin embargo, la evidencia es escasa, por lo cual en estos casos se inicia tratamiento combinado. ${ }^{44}$

En general, el porcentaje de erradicación es de $60 \%,{ }^{36}$ mayor en pacientes tratados con rituximab y otros inmunosupresores versus monoterapia, aunque, en algunas series, rituximab no fue mejor que los esteroides ( 77 versus $61 \%$ ) y el tiempo hasta la remisión fue mayor (mediana: 32 versus 64 días). La tasa de 
Tabla 4. Opciones de primera línea para tratamiento inmunosupresor

\begin{tabular}{|c|c|c|}
\hline $\begin{array}{l}\text { Inmunosupresión de } \\
\text { primera línea }\end{array}$ & Dosis recomendada & Comentario \\
\hline Esteroides & $\begin{array}{l}\text { - Prednisona } 1 \text { mg/kg/día. } \\
\text { - Dexametasona } 40 \text { mg/día/4-7 días.* }\end{array}$ & $\begin{array}{l}\text { - Poco probable que sea efectiva antes de } 3 \text { semanas } \\
\text { en pacientes con FVIII < } 1 \% \text { o con inhibidor }>20 \text { UB al } \\
\text { diagnóstico. } \\
\text { - Monitorear los eventos adversos. }\end{array}$ \\
\hline Esteroides y Ciclofosfamida & $\begin{array}{l}\text { - Esteroides en dosis similares a las de } \\
\text { arriba. } \\
\text { - Ciclofosfamida vía oral } 1-2 \mathrm{mg} / \mathrm{kg} / \mathrm{día} \text {. } \\
\text { - Ciclofosfamida intravenosa } 5 \mathrm{mg} / \mathrm{kg} \text { cada } \\
\text { 3-4 semanas. }{ }^{*}\end{array}$ & $\begin{array}{l}\text { - Respuestas más rápidas pero mayor posibilidad de } \\
\text { eventos adversos. } \\
\text { - Mayor posibilidad de respuestas completas. } \\
\text { - Monitorear mielosupresión. }\end{array}$ \\
\hline Esteroides y rituximab & $\begin{array}{l}\text { - Esteroides en dosis similares a las de } \\
\text { arriba. } \\
\text { - Rituximab intravenosa } 375 \mathrm{mg} / \mathrm{m}^{2} / \mathrm{semana} \\
\text { por } 4 \text { semanas. } \\
\text { - Rituximab } 100 \mathrm{mg} / \mathrm{semana} \text { por } 4 \\
\text { semanas. }\end{array}$ & $\begin{array}{l}\text { - Rituximab no se recomienda como monoterapia inicial a } \\
\text { menos que las demás terapias estén contraindicadas. }\end{array}$ \\
\hline \multicolumn{3}{|c|}{$\begin{array}{l}\text { El promedio de tiempo para la respuesta (FVIII > } 50 \text { \%) es } 5 \text { semanas. Los pacientes con FVIII < } 1 \% \text { al diagnóstico requieren periodos significativamente mayores para alcanzar } \\
\text { la remisión comparados con los pacientes con }>1 \% \text { de actividad de FVIII y requieren quizá más de la terapia combinada. La actividad del FVIII, así como la del inhibidor deben } \\
\text { monitorearse al menos una vez a la semana de acuerdo con la evolución clínica. La terapia se individualiza de acuerdo con la condición clínica del paciente, la enfermedad primaria, } \\
\text { los estados patológicos concomitantes y los factores pronósticos (FVIII < 1\%, inhibidor > } 20 \text { UB, presencia de anticuerpos anti-rpFVIII, etc.), si están disponibles. } \\
\text { "Evidencia pobre en hemofilia adquirida, pero con datos publicados en otras patologías autoinmunes. }\end{array}$} \\
\hline
\end{tabular}

recaída con rituximab parece menor que con esteroides más ciclofosfamida 0 esteroides solos $(4,14$ y $19 \%$, respectivamente). Hay evidencia positiva acerca del rituximab en dosis de $100 \mathrm{mg} / \mathrm{m}^{2} .{ }^{45}$ En la HA asociada con el embarazo, rituximab parece eficaz, aunque los casos corresponden a tratamientos luego de falla a esteroides y títulos altos de inhibidor. ${ }^{46}$

No existe evidencia de que algún inmunosupresor sea superior o para elegir alguno con base en el estado hemorrágico o título del inhibidor. La terapia de primera línea depende de evaluar la enfermedad de base y los eventos adversos probables. ${ }^{32}$ Las infecciones no son despreciables y pueden causar la muerte ${ }^{47}$ $y$, como en todo paciente con inmunosupresión prolongada, es necesaria la vigilancia inmunológica estrecha. Además de los inmunosupresores se usan azatioprina, ciclosporina, ciclofosfamida, micofenolato, 6-mercaptopurina, tacrolimus, cladribina, azatioprina y vincristina. La monoterapia con prednisona 0 combinada con ciclofosfamida se emplea con respuestas importantes. ${ }^{28,32,41}$ También se utilizan dosis altas de inmunoglobulina intravenosa. ${ }^{4} \mathrm{El}$ tratamiento combinado con quimioterápicos (ciclofosfamida, vincristina y prednisona) se indica si las demás opciones fallaron. En HA asociada con lupus eritematoso sistémico, la ciclosporina en dosis de $10-15 \mathrm{mg} /$ $\mathrm{kg} / \mathrm{día}$ parece útil. ${ }^{48}$

Existen dos tratamientos no globalmente aceptados ni ampliamente disponibles: inmunoadsorción e inmunotolerancia. En la primera, se hace pasar el plasma del paciente por una columna de sefarosa con proteína A de estafilococo adherida, capaz de eliminar auto-Ac; generalmente se utiliza en casos muy agudos y graves ${ }^{49}$ es un tratamiento caro, complicado, poco disponible y no se sabe si la erradicación es sostenida. La inmunotolerancia, útil en hemofilia hereditaria, es cara, su disponibilidad limitada y tampoco asegura la erradicación sostenida del inhibidor. La administración crónica y en dosis altas de FVIII induce regulación inmunitaria y aumento de la sensibilidad de la clona productora del auto-Ac al inmunosupresor, modulando la generación del auto-Ac. ${ }^{6}$ Quizá sea aplicable solo en HA recidivante y su uso amplio es improbable ${ }^{14,27}$

\section{Conclusiones}

El problema principal con la HA es el diagnóstico retardado por desconocimiento de la enfermedad. Son imperativas estrategias educativas a mediano y largo plazo para elevar la conciencia de esta entidad potencialmente curable en el médico, para incorporarla a sus diagnósticos. No es difícil sospechar la HA; bastan unas pruebas hemostáticas básicas e interpretar el cuadro clínico en poblaciones de riesgo. El siguiente reto es el laboratorio hematológico en el segundo y tercer nivel de atención, del cual debe enfatizarse su papel diagnóstico y la necesidad de afinar sus procesos para elevar la calidad de sus pruebas. Otro problema es la carencia de un registro nacional. Avanzamos, aunque insuficientemente, en hemofilia hereditaria (el 
subregistro es de $18 \%$ ); para HA solo contamos con la experiencia de los grandes centros de referencia. Desconocemos casi completamente la epidemiología en México; necesitamos datos que retroalimenten al sistema de salud para desarrollar políticas y guías terapéuticas realmente eficaces. Para el tratamiento, un reto es la fragilidad del paciente, sensible a inmunosupresión e infección secundarias, factores que pueden ocasionar la muerte por sepsis. Ya que los individuos con HA padecen varias comorbilidades (algunas requieren tratamiento antitrombótico), a quienes se indica medicación que genera trombina (agentes de puenteo, altas dosis de FVIII, DDAVP y antifibrinolíticos), algunos requerirán profilaxis antitrombótica.

\section{Bibliografía}

1. Collins PW, Chalmers E, Hart D, Jennings I, Liesner R, Rangarajan S, et al. Diagnosis and management of acquired coagulation inhibitors: a guideline from UKHCDO. Br J Haematol. 2013;162:758-773.

2. Oldenburg J, Zeitler H, Pavlova A. Genetic markers in acquired haemophilia. Haemophilia. 2010;16:41-45

3. Franchini M, Capra F, Nicolini N, Veneri D, Manzato F, Baudo F, et al. Drug- induced anti-factor VIII antibodies: a systematic review. Med Sci Monit. 2007;13:RA55-RA61.

4. Collins PW, Hirsch S, Baglin TP, Dolan G, Hanley J, Makris M, et al. Acquired hemophilia A in the United Kingdom: a 2-year national surveiIlance study by the United Kingdom Haemophilia Centre Doctors' Organisation. Blood. 2007;109:1870-1877.

5. Baudo F, Collins $P$, Huth-Kühne A, Lévesque H, Marco P, Nemes L, et al. Management of bleeding in acquired hemophilia A: results from the European Acquired Haemophilia (EACH2) Registry. Blood. 2012;120:39-46.

6. Reding MT, Lei S, Lei H, Green D, Gill J, Conti-Fine BM. Distribution of Th1- and Th2-induced anti-factor VIII IgG subclasses in congenital and acquired haemophilia patients. Thromb Haemost. 2002;88:568-575

7. Franchini M, Lippi G. Acquired haemophilia A. Adv Clin Chem. 2011; $54: 71-80$.

8. Huth-Kühne A, Baudo F, Collins P, Ingerslev J, Kessler CM, Lévesqueet H, et al. International recommendations on the diagnosis and treatment of patients with acquired hemophilia A. Haematologica. 2009;94:566-575.

9. Sakurai Y, Takeda T. Acquired hemophilia A: A frequently overlooked autoimmune hemorrhagic disorder. J Immunol Res. 2014;1:1-10.

10. Kruse-Jarres R, Kempton CL, Baudo F, Collin PW, Knoebl P, Leissinger CA, et al. Acquired hemophilia A: updated review of evidence and treatment guidance. Am J Hematol. 2017:92:695-705.

11. Windyga J, Chojnowski K, Klukowska A, Working Group on Haemostasis of the Polish Society of Haematology and Transfusion Medicine. Polish recommendations on the management of acquired haemophilia A. Med Prakt. 2011;10:42-51.

12. Zdziarska J, Musial J. Acquired hemophilia A: an underdiagnosed, severe bleeding disorder. Pols Arch Med. 2014:124:200-206.

13. Mingot-Castellano ME, Núñez R, Rodríguez-Martorell FJ. Acquired haemophilia: epidemiology, clinical presentation, diagnosis and treatment. Med Clin (Barc). 2017;148:314-322.

14. Franchini M, Lippi G. Acquired factor VIII inhibitors. Blood. 2008;112: 250-255.

15. Knoebl P, Marco P, Baudo F, Collins $P$, Huth-Kühne A, Nemes L, et al. Demographic and clinical data in acquired hemophilia $A$ : results from the European Acquired Haemophilia Registry (EACH2). J Thromb Haemost. 2012:10:622-631.

16. Tengborn L, Baudo F, Huth-Kühne A, Knoebl $P$, Lévesque $H$, Marco $P$. Pregnancy-associated acquired haemophilia $A$ : results from the European Acquired Haemophilia (EACH2) registry. BJOG. 2012;119:1529-1537.

17. Franchini M, Mannucci PM. Acquired haemophilia A: a 2013 update. Thromb Haemost. 2013;110:1114-1120.

18. Lulla RR, Allen GA, Zakarija A, Green D. Transplacental transfer of postpartum inhibitors to factor VIII. Haemophilia. 2010;16:14-17.

19. Collins PW, Percy CL. Advances in the understanding of acquired haemophilia A: implications for clinical practice. Br J Haematol. 2010;148:183-194.

20. Sahud MA. Semin Thromb Hemost. 2000;26:195-203.

21. Verbruggen B, Novakova I, Wessels H, Boezeman J, van den Berg M, Mauser-Bunschoten E. The Nijmegen modification of the Bethesda assay for factor VIII: C inhibitors: improved specificity and reliability. Thromb Haemost. 1995;73:247-251.
22. Werwitzke S, Geisen U, Nowak-Gottl U, Eichler H, Stephan B, Scholz U, et al. Diagnostic and prognostic value of factor VIII binding antibodies in acquired hemophilia A: data from the GTH-AH 01/2010 study. J Thromb Haemost. 2016;14:940-947.

23. Kasper CK, Aledort L, Aronsonet D, Counts R, Edson JR, van Eys J, et al. Proceedings: a more uniform measurement of factor VIII inhibitors. Thromb Diath Haem. 1975;34:612.

24. Pruthi RK, Nichols WL. Autoimmune factor VIII inhibitors. Curr Op Hematol. 1999;6:314-322.

25. Bird P. Coagulation in an agarose gel and its application to the detection and measurement of factor VIII antibodies. Br J Haematol. 1975;29:329-340.

26. Baudo F, Caimi T, de Cataldo F. Diagnosis and treatment of acquired haemophilia. Haemophilia. 2010;16:102-106.

27. Collins PW, Hirsch S, Baglin TP, Dolan G, Hanley J, Makris M, et al. Acquired haemophilia A in the United Kingdom: a 2-year national surveillance study by the United Kingdom Haemophilia Centre Doctors' Organisation. Blood. 2007;109:1870-1877.

28. Delgado J, Jiménez-Yuste $V$, Hernando-Navarro F, Villar A. Acquired haemophilia: review and meta-analysis focused on therapy and prognostic factors. Br J Haematol. 2003;121:21-35.

29. Toschi V, Baudo F. Diagnosis, laboratory aspects and management of acquired hemophilia A. Intern Emerg Med. 2010:5:325-333.

30. Zanon E, Milan M, Gamba G, Ambaglio C, Saggiorato G, Spiezia L, et al. Activated prothrombin complex concentrate (FEIBA®) for the treatment and prevention of bleeding in patients with acquired haemophilia: a sequential study. Thromb Res. 2015;136:1299-1302.

31. Kruse-Jarres R, St-Louis J, Greist A, Shapiro A, Smith H, Chowdary P, et al. Efficacy and safety of OBI-1, an antihaemophilic factor VIII (recombinant), porcine sequence, in subjects with acquired haemophilia $A$. Haemophilia. 2015;21:162-170.

32. Collins PW. Treatment of acquired hemophilia A. J Thromb Haemost. 2007:5:893-900.

33. Dunkley SM, Yang K. The use of combination FEIBA and rFVIla bypassing the-rapy, with TEG profiling, in uncontrollable bleeding associated with acquired haemophilia A. Haemophilia. 2009;15:828-830.

34. Goudemand J. Treatment of bleeding episodes occurring in patients with acquired haemophilia with FEIBA: the French experience. Haemophilia. 2004;10:PO14.

35. Hay CR, Negrier C, Ludlam CA. The treatment of bleeding in acquired haemophilia with recombinant factor VIla: a multicentre study. Thromb Haemost. 1997;78:1463-1467.

36. Sumner MJ, Geldziler BD, Pedersen M, Seremetis S. Treatment of acquired haemophilia with recombinant activated FVII: a critical appraisal. Haemophilia. 2007;13:451-461

37. Tiene A, Amano K, Ma A, Arkhammar P, Según SB, Rosholm A, et al The use of recombinant activated factor VII in patients with acquired haemophilia. Blood Rev. 2015;29:S19-S25.

38. Zeng $\mathrm{Y}$, Zhou R, Duan X, Long D, Yang S. Interventions for treating acute bleeding episodes in people with acquired hemophilia A. Cochrane Database Syst Rev. 2014;8:CD010761.

39. Nicolaides AN, Breddin HK, Carpenter P, Coccheri S, Conard J, de Stefano V, et al. Thrombophilia and venous thromboembolism. International consensus statement. Guidelines according to scientific evidence. Int Angiol. 2005;24:1-26

40. Sakurai $Y$, Takeda T. Acquired hemophilia A: a frequently overlooked autoimmune hemorrhagic disorder. J Immunol Res. 2014:1:1-10.

41. Green D, Rademaker AW, Briët A. A prospective, randomized trial of prednisone and cyclophosphamide in the treatment of patients with factor VIII autoantibodies. Thromb Haemost. 1993;70:753-757.

42. Sperr WR, Lechner K, Pabinger I. Rituximab for the treatment of acquired anti-bodies to factor VIII. Haematologica. 2007;92:66-71.

43. Field JJ, Fenske TS, Blinder MA. Rituximab for the treatment of patients with very high-titre acquired factor VIII inhibitors refractory to conventional chemotherapy. Haemophilia. 2007;13:46-50.

44. Aggarwal A, Grewal R, Green RJ, Boggio L, Green D, Weksler BB, et al. Rituximab for autoimmune haemophilia: a proposed treatment algorithm. Haemophilia. 2005;11:13-19.

45. Wermke M, von Bonin M, Gehrisch S, Siegert G, Enhinger G, Plazbeker U. Successful erradication of acquired factor-VIII-inhibitor using single low-dose rituximab. Haematologica. 2010;95:521-52.

46. Bonfanti C, Crestani S, Frattini F, Sissa C, Franchini M. Role of rituximab in thetreatment of postpartum acquired haemophilia $\mathrm{A}$ : a systematic review of the literature. Blood Transfus. 2015;13:396-400.

47. Tanaka I, Amano K, Taki M, Oka T, Sakai M, Shirahata A, et al. A 3-year consecutive survey on current status of acquired inhibitors against coagulation factors in Japan: analysis of prognostic factors. Jap J Thromb Hemostas. 2008;19:140-153.

48. Lacroix-Desmazes S, Bayry J, Misra N, Horn MP, Villard S, Pashov A, et al. The prevalence of proteolytic antibodies against factor VIII in hemophilia A. N Eng J Med. 2002;346:662-667.

49. Knöbl P, Derfler K. Extracorporeal immunoadsorption for the treatment of haemophilic patients with inhibitors to factor VIII or IX. Vox Sango. 1999;77:57-64. 